teratogenic factors playing a role. They can occur isolated or in association with multiple syndromes. Although common, there are few published studies focusing on the subject The aim of this study is to describe the ocular anomalies found within a population of patients with OFC.

Methods A retrospective analysis of medical reports of patients that attended or are attending the clinic of Clef Lip and Palate multidisciplinary group at Centro Hospitalar São João in Porto - Portugal over a period of 27 years between January 1992 and December 2018. OFC types were classified according to Spina classification: cleft lip (CL), cleft lip and palate (CLP), isolated cleft palate (CP) and atypical clefts (AC).

Results In total, 617 patients were included from which 106 (17.2\%) had ocular anomalies, which were the most frequent type of anomaly within the study group; $58.8 \%$ were females. The most frequent ocular findings were myopia (30\%), strabismus (21\%) and astigmatism (14\%). Other relevant findings were the presence of coloboma in $8.5 \%$, short palpebral fissure in $8.5 \%$ and microphthalmia in $6.6 \%$. Ocular anomalies were mostly related to CP (61.7\%) followed by CLP $(26.2 \%)$ and CL (10.3\%). From the total sample of 106 patients, 70\% had an identified syndrome, the most common being the Pierre Robin Sequence or Syndrome. Other systems were affected as well with $38 \%$ of patients showing other craniofacial anomalies and 39\% cardiovascular anomalies.

Conclusions Ocular anomalies are frequent in the general population namely refractive errors such as myopia. Despite that, an important number of syndromes and congenital malformations are associated to ocular anomalies and OFC are among those. Although a significant number of patients with ocular anomalies were syndromic, not all OFC patients had other associated malformations. Therefore, awareness of the possible ocular malformations associated with OFC could provide a quick diagnosis and management of the affected patients.

\section{P128 A RETROSPECTIVE AUDIT OF MICROARRAY TESTING PERFORMED IN THE DEVELOPMENTAL CLINIC AT A TERTIARY PAEDIATRIC CENTRE}

${ }^{1}$ Thomas McGrath*, ${ }^{1}$ Stephanie Kelly, ${ }^{2}$ Louise Gallagher, ${ }^{1}$ Emma Curtis. ${ }^{1}$ Children's Health Ireland, Tallaght University Hospital, Dublin, Ireland; '2Psychiatry, Trinity Centre for Health Sciences, St James's Hospital, Dublin, Ireland

\subsection{6/archdischild-2019-epa.483}

Background Array comparative genomic hybridisation (microarray) testing is a high resolution genetic test which detects microscopic and sub-microscopic chromosomal aberrations ${ }^{1}$. In recent years, it has become the first line genetic investigation in children presenting to paediatric services with unexplained global developmental delay (DD), intellectual disability (ID) and autism spectrum disorders (ASD) 1,2 . Previous studies have shown that the diagnostic yield of microarray testing is between 15 and $20 \%{ }^{2}$.

Objectives The aim of this retrospective audit was to investigate the use of microarray testing of children presenting to a specialist developmental clinic, comparing the clinical indication against proposed national and international guidelines, ${ }^{1,2}$, as well as, documenting abnormal results identified and their correlation with clinical phenotype with a view to refining the screening protocol.

Methods A total of 164 microarray tests performed on patients presenting to the clinic in 2017 were reviewed. The clinical indication prompting testing was documented. The overall number of copy number variants in the population studied was determined and each $\mathrm{CNV}$ was characterised and its clinical significance assessed.All results had previously been disclosed to patients and families and all data anonymised.

Results The most common clinical indications for the 164 microarray tests included ASD, DD, and speech and language delay accounting for 99, 26, and 11 cases respectively.Other less common indications included gross motor delay, ID, and developmental coordination disorder $(<10)$.A total of 42 copy number variants were identified in 36 (29\%) patients. Of the 42, $16(9.8 \%)$ were associated with a previously documented genetic abnormality on the Online Mendelian Inheritance in Man database, 3 (1.8\%) were variations of unknown significance and 23 (14\%) were benign copy number variants (BCNV).

Of the 99 children with autism, 19 (19.2\%) demonstrated a genetic aberration $8(8.1 \%)$ of which were previously described on the OMIM database, 1 (1\%) was a variation of unknown significance and $8(8.1 \%)$ were BCNVs.

Conclusion Advances in genetic testing have transformed investigation in children with developmental abnormalities and there is an expectation that such investigations will be performed. Within the group of children with a diagnosis of autism, the explanation and understanding of the abnormality can be difficult. This presentation will explore further the clinical phenotype and genetic results of the children in this study with discussion regarding guidance for genetic testing in this patient group.

\section{P129 RISK FACTORS FOR DEVELOPMENTAL DYSPLASIA OF THE HIP: 10-YEAR EXPERIENCE}

${ }^{1}$ Bahar Kural*, ${ }^{2}$ Esra Devecioglu, ${ }^{2}$ Gülbin Gökçay, ${ }^{2}$ Tijen Eren, ${ }^{3}$ Pınar Yılmazbaş, ${ }^{4}$ Irfan Gökçay. 'Department of Pediatrics,Bakırkoy Research and Training Hospital, Istanbul, Turkey; ${ }^{2}$ Department of Social Pediatrics, Institute of Child Health, Istanbul University, , Istanbul, Turkey; ${ }^{3}$ Department of Pediatrics, Okmeydanı Research and Training Hospital, Istanbul, Turkey; ${ }^{4}$ Department of Orthopedics, Okmeydanı Research and Training Hospital, Istanbul, Turkey

\subsection{6/archdischild-2019-epa.484}

Aim Risk based screening for developmental dysplasia of the hip $(\mathrm{DDH})$ with ultrasound is common. However, risk factors vary from one country to the other since data are insufficient to give clear recommendations. We aimed to evaluate the risk factors for developmental dysplasia of the hip (DDH).

Methods In this retrospective case-control study; the health records of all children, who were followed up between 2004 and 2014 at a well-child unit were investigated for the diagnosis of DDH. Of 9758 children, 57 children were found to have abnormal ultrasonographic findings and these constituted the case group. As the control group, healthy 228 children who matched the case children in birth months were selected. Two groups were compared for the risk factors.

Results A total of 19516 hips of 9758 children were examined for DDH. 97 hips of 57 children were found to have abnormal ultrasonographic findings (according to Graf classification). When the two groups were compared; breech presentation, multiple pregnancy and torticollis were identified as risk factors. The female sex was also found to have a significantly high prevalence among the children in the case group. Limited hip abduction, positive Ortolani and Barlow signs were important clinical findings in the case group. 
Conclusion According to our findings; breech presentation, female sex, torticollis and multiple pregnancy were found to be the risk factors of this disorder. Infants with these risk factors should be investigated carefully for $\mathrm{DDH}$.

\section{P130 FIFTEEN-MINUTE CONSULTATION: THE INFANT WITH A SMALL HEAD}

${ }^{1}$ Joshua Alexander*, ${ }^{1}$ Nusrat Bholah, ${ }^{1}$ Naomi Okigbo, ${ }^{1}$ Ruth Devlin, ${ }^{1}$ Aisling Betts, ${ }^{1}$ Jack Healy, ${ }^{1}$ Sarah Higgins, ${ }^{1,2}$ Judith Meehan, 1,2,3,4Eleanor Molloy. 'Paediatrics, Trinity College Dublin, the University of Dublin, Dublin, Ireland; ${ }^{2}$ Tallaght University Hospital, Dublin, Ireland; ${ }^{3}$ Paediatrics, Coombe Women and Infants University Hospital, Dublin, Ireland; ${ }^{4}$ Neonatology, Our Lady's Children's Hospital, Dublin, Ireland

\subsection{6/archdischild-2019-epa.485}

Aims Microcephaly is a feature of multiple diseases, and has a vast array of aetiologies. Thus, identifying this condition clinically is only the start of establishing a diagnosis. Early assessment, analysis, and identification is vital to aiding the patient and family in managing the microcephaly itself, as well as future complications that may arise as a result. We aim to provide general practitioners, paediatricians and other health professionals, with a framework for initial approach to a patient presenting with a small head.

Methods An electronic review of literature on databases PubMed, Google Scholar, and Science Direct was performed searching Title/Abstract with the terms: [Microcephaly AND (assessment OR consultation OR examination)]. Previously published guidelines with information on initial approach to the microcephalic patient were also used in production of the proposed protocol.

Results We devised a protocol which helps clinicians to firstly diagnose microcephaly, and then separate primary microcephaly from secondary microcephaly. We then outlined potential investigations that should be undertaken to identify any underlying conditions. For primary microcephaly, specialised cytogenetic studies and gene sequencing and use of microarray can help to diagnose syndromes resulting in microcephaly. ${ }^{[i]}$ Secondary microcephaly is investigated through a comprehensive viral screen and range of biochemical blood tests to identify underlying causes. ${ }^{[i i]}$ The above methods are often combined with various imaging modalities, commonly MRI brain.

Conclusion As microcephaly is a clinical finding rather than a disease, a careful clinical history and examination are the cornerstones of the consultation, with findings guiding further investigations.

\section{REFERENCE}

1. Woods CG, Parker A. Investigating microcephaly. Archives of Disease in Childhood. 2013;98(9):707-713.

2. Michels T, Morris M, Myers T. Microcephaly. [internet]. 2009. Available from: https://core.ac.uk/download/pdf/62772193.pdf

\section{P131 'SEVERE NEUROLOGICAL IMPAIRMENT' - DOES THE TERM DEFINE AN AGREED COHORT FOR STUDY?}

1,2John Allen*, 1,2,3,4Eleanor Molloy, 1,2Denise McDonald. ${ }^{1}$ Trinity College Dublin, Dublin, Ireland; 'Tallaght University Hospital, Dublin, Ireland; ${ }^{3}$ Coombe Women and Infant's University Hospital, Dublin, Ireland; ${ }^{4}$ Our Lady's Children's Hospital Crumlin, Dublin, Ireland

10.1136/archdischild-2019-epa.486
Background Severe Neurological Impairment (SNI) is a term commonly used in the medical literature. There is no agreed definition of SNI. This limits opportunities for research into healthcare needs, treatment opportunities, resource planning and outcome. We reviewed the literature to establish consistency of use of the term and to place it in the context of other commonly employed terms used to describe children with severe, complex medical needs.

Methods A review of the literature was performed in October 2018. We searched the electronic database of PubMed and the Cochrane library. The search term 'Severe Neurological Impairment' was used. Animal studies were excluded but no other constraints were placed on the literature search. Fortytwo articles were included for full-text analysis. Definitions were analysed for common themes. Data were entered into an electronic spreadsheet and descriptive statistics were used determine frequency of the themes included in the various definitions.

Results Twenty-three of the papers reviewed included a definition of SNI, where the authors stated their interpretation of the use of the term SNI. In the other papers reviewed, the profile of the patients included by the authors provides insight into their understanding of the term. Motor impairment, intellectual disability, communication difficulties and increased care needs were included in the definition in $80 \%, 70 \%, 30 \%$ and $13 \%$ of papers respectively. Dependence on others for decision-making, chronicity and distinction between disorders of the central nervous system and peripheral nervous system were less frequently included.

Discussion This review demonstrates inconsistency in the use of the term Severe Neurological Impairment in the literature. Several other definitions exist to describe children with severe and complex medical conditions including Children with Special Healthcare Needs and Children with Medical Complexity. These definitions are often broad and include children with a wide variety of difficulties. Given their unique health and social care needs in the context of neurological impairment, an agreed definition distinguishing SNI from others would be helpful as a starting point to assess need and plan services.

Conclusions We have performed a comprehensive review of the literature and have shown that there is a wide variation in the use of the term Severe Neurological Impairment. A consensus-based definition of this term would be useful to facilitate future research.

\section{P132 EARLY FEEDING PROBLEMS AND ASSOCIATED FACTORS IN PREMATURE INFANTS}

${ }^{1}$ Ayşe Nur Coşkun*, ${ }^{2}$ Pınar Zengin Akkuş, ${ }^{2}$ Evin IIlter Bahadur, ${ }^{3}$ Hasan Tolga Çelik, ${ }^{2}$ Elif Nursel Özmert. ${ }^{1}$ Hacettepe University Faculty of Medicine, Department of Pediatrics, ANKARA, Turkey; ${ }^{2}$ Hacettepe University Faculty of Medicine, Department of Developmental an Behavioral Pediatrics, ANKARA, Turkey; ${ }^{3}$ Hacettepe University Faculty of Medicine, Department of Neonatology, ANKARA, Turkey

\subsection{6/archdischild-2019-epa.487}

Aim The aim of this study was to determine the frequency of feeding problems in premature infants and to investigate the relationship between growth, development and maternal depression.

Methods The study included 58 premature infants who were admitted to the Developmental Pediatrics Clinic between February-August 2018. Feeding problems obtained from the mothers by a questionnaire were classified as problems with oral- 